\title{
Intervenções não farmacológicas no tratamento da neuropatia periférica induzida pela quimioterapia
}

\author{
Non-pharmacological interventions in the treatment of peripheral neuropathy induced by \\ chemotherapy
}

Intervenciones no farmacológicas en el tratamiento de la neuropatía periférica inducida por la quimioterapia

\begin{abstract}
Gabriella Carolayne Fernandes Henrique ${ }^{1 *}$, Themístoclys Thesko Correia Ferreira², Ana Patrícia de Arruda Silva ${ }^{1}$, Izadora Bezerra Varjão de Melo Santos ${ }^{1}$, Elange do Carmo Fernandes de Oliveira1 e Valdir Lucas Marques de Souza1.
\end{abstract}

\section{RESUMO}

Objetivo: Verificar na literatura científica a utilização dos recursos não farmacológicos no tratamento da neuropatia periférica induzida pela quimioterapia (NPIQ). Métodos: Este estudo trata-se de uma revisão narrativa, realizada nas bases de dados MEDLINE/PUBMED, LILACS, SCIELO e PEDRO, utilizando como critérios de inclusão: ensaio clínico, sem restrição temporal, nas línguas portuguesa e inglesa. Os critérios de exclusão foram: estudos que associassem a neuropatia a lesões cirúrgicas e pesquisas que incluíam tratamentos não farmacológicos fora do âmbito da Fisioterapia. Resultados: Foram analisados quatro artigos que descreveram as intervenções não farmacológicas para o tratamento da NPIQ através do uso de terapia manual, massoterapia, treinamento visual de equilíbrio do feedback do computador e um programa que abrange treinos de equilíbrio e coordenação, exercícios aeróbicos e de fortalecimento muscular global. Onde pôde observar os benefícios dos respectivos tratamentos nos pacientes com NPIQ, como redução do quadro álgico e da parestesia, melhora do equilíbrio e força, devolvendo assim a funcionalidade e a percepção de qualidade de vida. Conclusão: A NPIQ traz sérias complicações, ocasionando disfunções e afetando suas atividades de vida diária. Existem pesquisas com recursos não farmacológicos para o tratamento e controle da NPIQ. Porém são escassos estudos que comprovem a eficácia dessas terapias.

Palavras-chave: Câncer, Neuropatia periférica induzida pela quimioterapia, Recursos terapêuticos.

\begin{abstract}
Objective: To verify in the scientific literature the use of non-pharmacological resources in the treatment of peripheral neuropathy induced by chemotherapy (NPIQ). Methods: This study is a narrative review carried out in the MEDLINE / PUBMED, LILACS, SCIELO and PEDRO databases, using as inclusion criteria: clinical trial, without temporal restriction, in the Portuguese and English languages. Exclusion criteria were: studies associating neuropathy with surgical injuries and investigations that included non-pharmacological treatments outside the scope of Physical Therapy. Results: Four articles describing non-pharmacological interventions for the treatment of NPIQ were analyzed through the use of manual therapy, massage therapy, visual training of computer feedback balance and a program that includes balance and coordination training, aerobic and strengthening exercises muscular activity. Where he could observe the benefits of the respective treatments in patients with NPIQ, such as reduction of pain and paresthesia, improvement of balance and strength, thus restoring the functionality and perception of quality of life. Conclusion: The NPIQ brings serious complications, causing dysfunctions and affecting its activities of daily living. There are researches with non-pharmacological resources for the treatment and control of NPIQ. However, there are few studies that prove the efficacy of these therapies.
\end{abstract}

Keywords: Cancer, Peripheral neuropathy induced by chemotherapy, Therapeutic resources.

\footnotetext{
${ }^{1}$ Acadêmico de Fisioterapia do Centro Universitário Brasileiro - UNIBRA. *E-mail: gabriella.carolayne@hotmail.com ${ }^{2}$ Fisioterapeuta, Especialista em Fisioterapia em Oncologia, Docente do curso de Fisioterapia da UNIBRA.
} 


\section{RESUMEN}

Objetivo: Verificar en la literatura científica la utilización de los recursos no farmacológicos en el tratamiento de la neuropatía periférica inducida por la quimioterapia (NPIQ). Métodos: Este estudio es una revisión narrativa realizada en MEDLINE / PubMed, LILACS y SCIELO PETER utilizando los siguientes criterios de inclusión: ensayos clínicos, sin restricción de tiempo, en Inglés e Inglés. Los criterios de exclusión fueron: estudios que asociar la neuropatía a lesiones quirúrgicas e investigaciones que incluían tratamientos no farmacológicos fuera del ámbito de la Fisioterapia. Resultados: Se analizaron cuatro artículos que describieron las intervenciones no farmacológicas para el tratamiento de la NPIQ a través del uso de terapia manual, masoterapia, entrenamiento visual de equilibrio de la retroalimentación del ordenador y un programa que abarca entrenamientos de equilibrio y coordinación, ejercicios aeróbicos y de fortalecimiento muscular global. En los pacientes con NPIQ, como la reducción del cuadro álgico y de la parestesia, mejora el equilibrio y la fuerza, devolviendo así la funcionalidad y la percepción de calidad de vida. Conclusión: La NPIQ trae serias complicaciones, ocasionando disfunciones y afectando sus actividades de vida diaria. Existen investigaciones con recursos no farmacológicos para el tratamiento y control de la NPIQ. Pero son escasos estudios que demuestren la eficacia de esas terapias.

Palabras clave: Cáncer, Neuropatía periférica inducida por la quimioterapia, Recursos terapéuticos.

\section{INTRODUÇÃO}

Câncer ou Neoplasia maligna é uma lesão constituída por proliferação celular anormal, descontrolada e autônoma, em geral com perda ou redução de diferenciação celular, em consequência de alterações em genes e proteínas que regulam a multiplicação e diferenciação das células, desenvolvendo a capacidade de invasão para outros tecidos e órgãos, tornando-se uma condição mortal, caso não seja instituído um tratamento adequado (KLECKNER IR, et al., 2018).

A sua prevalência no mundo tem aumentado de maneira significativa no último século, entre os tumores mais incidentes no mundo encontra-se câncer de mama sendo mais comum no sexo feminino, câncer de próstata sendo mais incidente no sexo masculino e câncer de colón e reto em ambos os sexos, sendo mais prevalente em mulheres. Entre as justificativas do aumento de novos casos das doenças oncológicas, destacam-se: o aumento da industrialização e da urbanização, que leva a grande mudança no estilo de vida, como o aumento do tabagismo, alcoolismo, obesidade, sedentarismo, entre outros (XAVIER ATF, et al., 2009).

Existem vários tipos de tratamento como cirurgia, radioterapia, hormonioterapia, imunoterapia e a quimioterapia que é a mais recomendada para diversos tipos de câncer, porém a sua utilização pode trazer complicações, devido aos seus efeitos colaterais, dentre elas está a neurotoxicidade, também conhecida como a neuropatia periférica induzida pela quimioterapia (NPIQ), que ocorre devido à degeneração ou disfunção dos nervos periféricos, em seu trajeto da medula espinhal até a periferia, podendo ocasionar alterações motoras, sensitivas ou autonômicas. Sua incidência na população geral varia de $4 \%$ a $5 \%$, sendo relacionado com tipo de quimioterápico utilizado, a dose e o esquema de administração (TONEZZER T, et al., 2016).

A NPIQ pode gerar um impacto negativo na qualidade de vida dos acometidos, pois essa alteração apresenta diversos sintomas de difícil controle, entre eles: dor, parestesia, dormência e fraqueza muscular, sendo uma das principais causas da redução da dose do quimioterápico ou em casos mais graves interrupção do tratamento (BEIJERS AJ, et al., 2015).

Segundo a Organização Europeia para Pesquisa e Tratamento do Câncer (EORTC), existem alguns instrumentos validados para a avaliação dos sintomas da NIPQ, sendo o mais o utilizado o Questionário Quality of Life Questionnaire (QLQ) -CIPN20 que contém 3 sub-escalas que avaliam sintomas sensoriais, motores e sintomas autonômicos onde o escore varia de 0 a 100 onde a maior pontuação representa maiores intensidades dos sintomas desta complicação. Também são utilizados outros métodos de avaliação, como o exame neurofisiológico, que incluiu estudos de condução nervosa e/ou testes sensorial quantitativo, exame neurológico, sendo realizados sozinhos ou em combinação (ORTEGA MHD, et al., 2013). 
Quando a NPIQ é diagnosticada e tratada os resultados são satisfatórios, existem dois fármacos que podem ser utilizados no tratamento clinico, são eles: a gabapentina, droga da classe dos anticonvulsivantes, porém, muito utilizada no tratamento da dor neuropática e a pregabalina, fármaco pertencente à classe terapêutica de analgésicos, miscelânea e anticonvulsivante. Ambas as drogas possuem como ação principal a modulação das transmissões nervosas dolorosas ao sistema nervoso central, reduzindo a atividade excitatória responsável pela dor neuropática. Elas são usadas como monoterapia de primeira linha para o alívio da dor em adultos com neuropatia periférica, e dor neuropática associada com lesão da medula espinal (ORTEGA MHD, et al., 2013).

Há na literatura a indicação de tratamentos não farmacológicos que são realizados com o objetivo potencializarem o controle dos sintomas da NPIQ e tratar possíveis alterações funcionais. Esses tratamentos vêm se destacando como de fundamental importância para a recuperação dos indivíduos que desenvolve essa complicação, apresentando como principais objetivos terapêuticos, entre eles: o controle ou diminuição do quadro álgico, a restauração da força muscular e sensibilidade, favorecendo a reabilitação motora e funcional, proporcionando ao paciente um maior bem-estar e melhoria na qualidade de vida (TONEZZER T, et al., 2016).

Diante do exposto, o objetivo deste artigo é explorar na literatura científica a utilização dos recursos não farmacológicos no tratamento e controle da NPIQ.

\section{MÉTODOS}

O presente estudo trata-se de uma revisão narrativa, que segundo Vosgerau DSR e Romanowski JP (2014), consiste em organizar, esclarecer e resumir as principais obras existentes, bem como fornecer citações completas abrangendo o aspecto de literatura relevante em uma determinada temática. Foi realizado nos meses de outubro de 2018 a março de 2019 através de pesquisas em artigos científicos publicados sem restrição temporal, nas línguas portuguesa e inglesa, nas bases de dados: Medical Literature Analysis and Retrieval System Online (MEDLINE/PUBMED), Literatura Latino-Americana e do Caribe em Ciências da Saúde (LILACS), Scientific Electronic Library Online (SCIELO) e Physotherapy Evidence Database (PEDRO).

Para a busca de artigos foram utilizados os Descritores em Ciências da Saúde (DECS): "quimioterapia combinada", "neoplasia", "doenças do sistema nervoso periférico" e "fisioterapia". Para pesquisa na língua inglesa na base de dados MEDLINE/PUBMED e PEDRO foram empregados os termos do Medical Subject Heading (MESH): "Combined chemotherapy", "neoplasia", "peripheral nervous system diseases" and "physiotherapy". Para ampliar as buscas, foi utilizado o Operador Booleano "and".

Quanto aos critérios de elegibilidade, foram incluídos estudos do tipo ensaios clínicos realizados na população humana, na faixa etária adulta.

Já os critérios de exclusão adotados nesta revisão foram: estudos que associassem a neuropatia a lesões cirúrgicas, assim como, pesquisas que incluíam tratamentos não farmacológicos fora do âmbito da Fisioterapia e artigos duplicados nas bases de dados pesquisadas.

Depois de empregadas as estratégias de buscas, os estudos selecionados foram avaliados através da leitura dos títulos e resumos. Em seguida, as pesquisas que apresentavam estrita relação com o tema estudado, foram lidas na íntegra com o objetivo de compor esta revisão narrativa.

\section{RESULTADOS}

Para a realização desta revisão foi definida a utilização de estudos originais, que utilizassem na sua metodologia apenas o tratamento não medicamento para NPIQ.

Os artigos identificados pela busca foram selecionados e avaliados segundo os critérios de elegibilidade do diagrama desenvolvido pelo Preferred Reporting Items for Systematic Reviews and Meta-Analyses (The PRISMA Statement) (Fluxograma 1). 
Fluxograma 1 - The Prisma Statement.
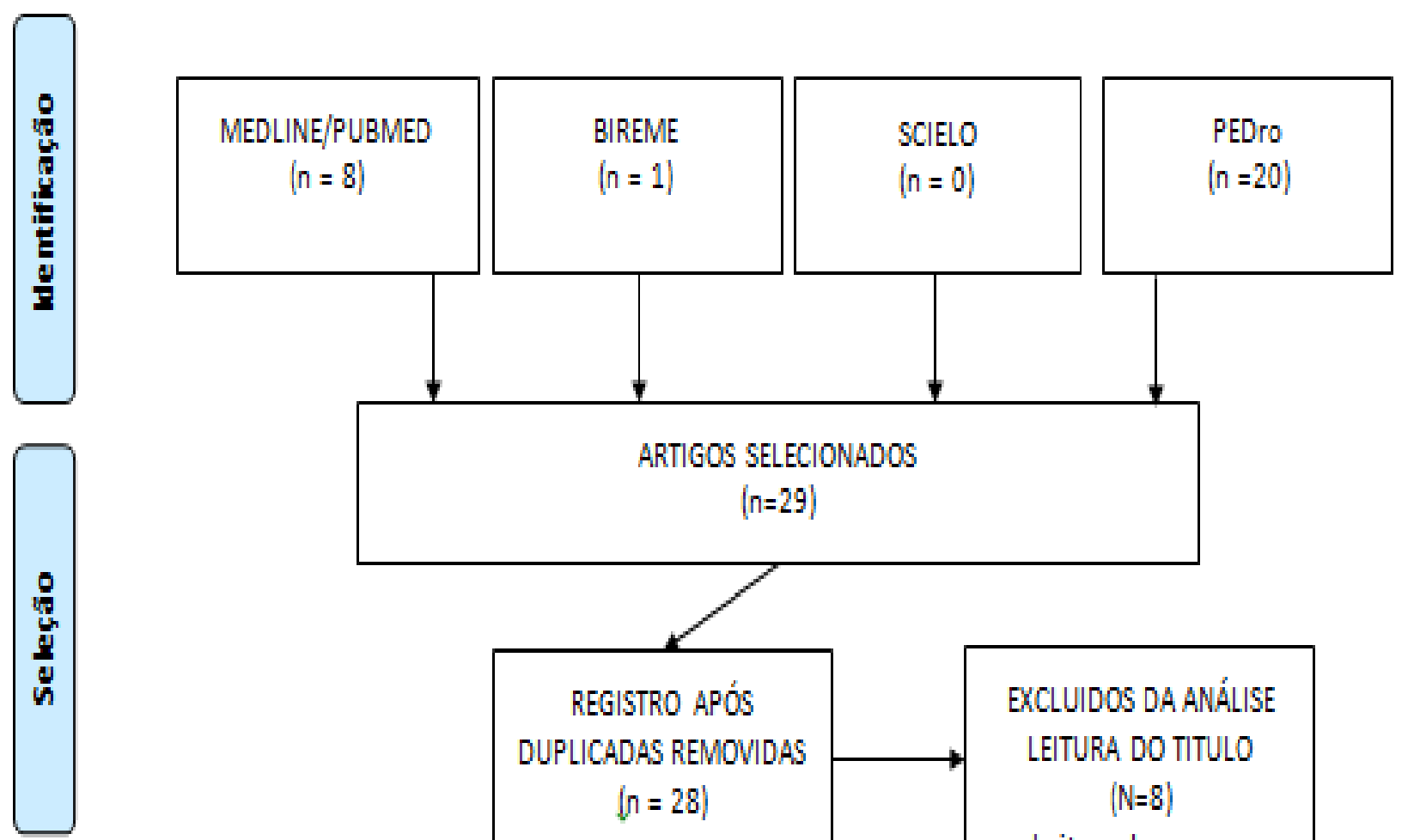

ARTIGOS SELECIONADOS

$(n=29)$
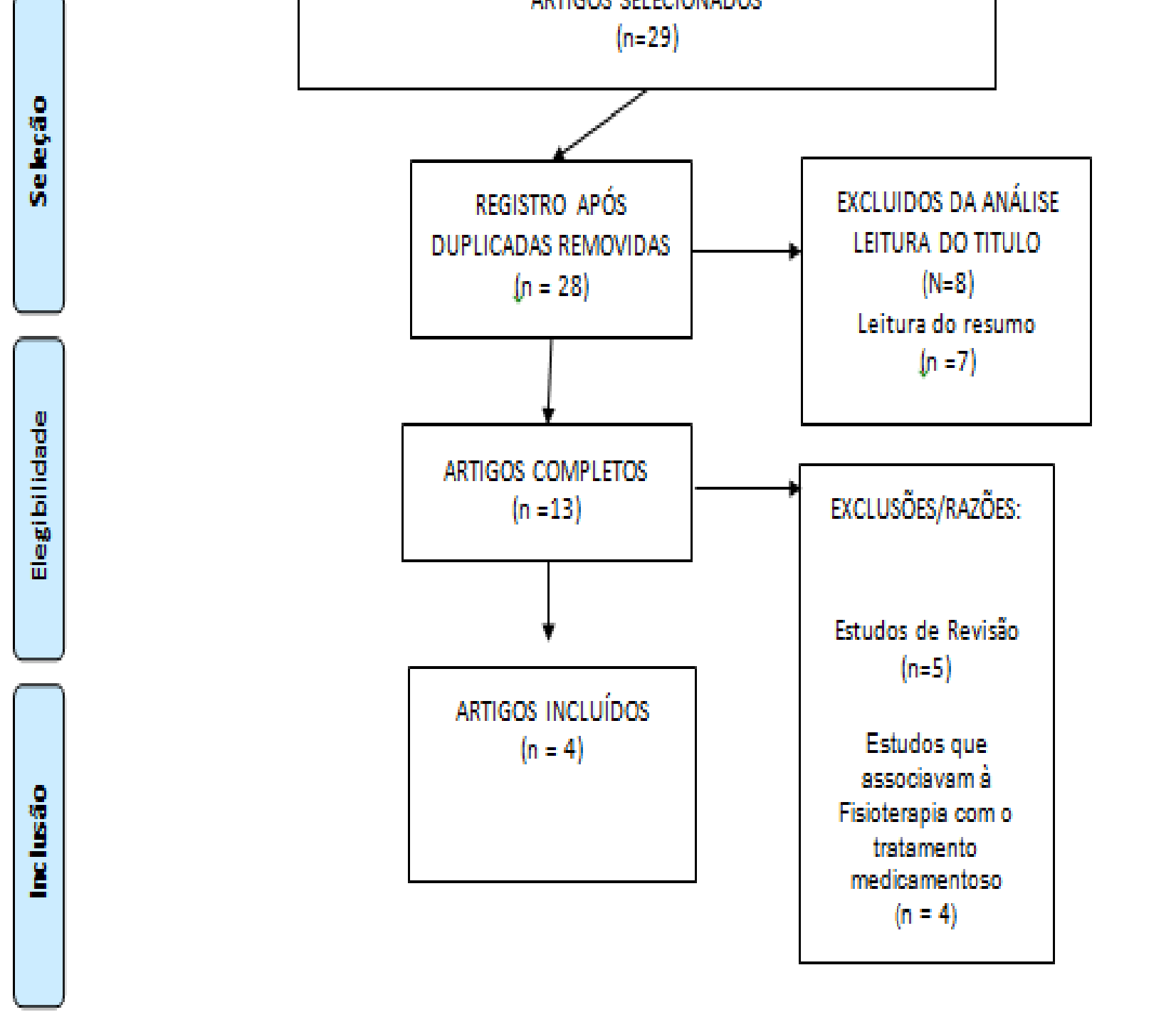

Fonte: Desenvolvido pelos autores Henrique GCF, et al., 2019.

Deste modo, o presente trabalho contou com a análise de quatro artigos, destes, dois estudos (50\%) tratava-se de ensaios clínicos randomizados, ambos publicados em 2017, um estudo (25\%) era do tipo estudo piloto, publicado em 2016 e o último estudo era o relato de caso, publicado em 2011. As informações contendo as características destes trabalhos (autor/ano de publicação, tipo de estudo, amostra, objetivo do estudo e resultados), estão descritas em uma tabela elaborada pelos autores desta revisão (Tabela 1). 
Tabela 1 - Síntese dos Resultados dos artigos selecionados.

\begin{tabular}{|c|c|c|c|c|}
\hline Autor/Ano & Tipo de Estudo & Amostra & Objetivo & Resultados \\
\hline $\begin{array}{l}\text { ZIMMER P et al., } \\
2017\end{array}$ & $\begin{array}{l}\text { Ensaio clínico } \\
\text { randomizado }\end{array}$ & $\begin{array}{l}30 \quad \text { participantes, } \\
\text { idosos, } 80 \text { anos ou } \\
\text { mais, } \\
\text { neoplasia } \\
\text { colorretal, com } \\
\text { ambos os sexos. }\end{array}$ & $\begin{array}{l}\text { Investigar a } \\
\text { influência de um } \\
\text { programa de } \\
\text { exercícios } \\
\text { supervisionados } \\
\text { como tratamento } \\
\text { adicional para } \\
\text { NPIQ. }\end{array}$ & $\begin{array}{l}\text { Os sintomas neuropáticos } \\
\text { mantiveram-se estáveis no Gl } \\
\text { ao longo do tempo, enquanto a } \\
\text { NPIQ piorou significativamente } \\
\text { no GC. O grupo intervenção } \\
\text { participou de um programa de } \\
\text { exercícios supervisionados de } \\
\text { oito semanas, onde houve a } \\
\text { melhora significativa na função } \\
\text { de força e equilíbrio. }\end{array}$ \\
\hline
\end{tabular}

SCHONSTEINER 131 participantes, Avaliar a Não houve diferença Ensaio clínico adultos e idosos, vibração do significativa na proporção de SS et al., 2017 randomizado idade mediana foi corpo inteiro pacientes com TRC normal de 60 (intervalo de (WBV) no $(<10 \mathrm{~s})$ no acompanhamento 24 a 71) anos. 44 contexto de um entre o GI (68\%) e GC (56\%) (p participantes tinham neoplasias hematológicas e 87 tumores sólidos.

programa integrado, incluindo massagem, mobilização passiva exercícios físicos em indivíduos com NPIQ. $=0,20)$.

Todos os pacientes experimentaram menos sintomas e dor $(p<0,001)$ e melhoraram a TRC $(p<0,001)$ ao longo do tempo. A WBV foi significativamente associada a uma maior redução do tempo necessário para TRC $(p=0,02)$ e melhorou significativamente - limiar de detecção de calor comparando a avaliação inicial com a de acompanhamento ( $p$ $=0,02)$.

CAMMISULI $S$ et Estudo Piloto al., 2016
Sete pacientes (1

homem e 6 mulheres), idades de 45 a 76 anos, com diagnóstico de: carcinoma mamário (3), linfoma nãoHodgkin carcinoma bexiga carcinoma ovário carcin (1).

1 paciente do sexo CUNNINGGAM Relato de caso JE et al., 2011 masculino, com grau 2 de NPIQ subsequente a um tratamento prévio com docetaxel e cisplatina para

a Fase III de adenocarcinoma esofágico.

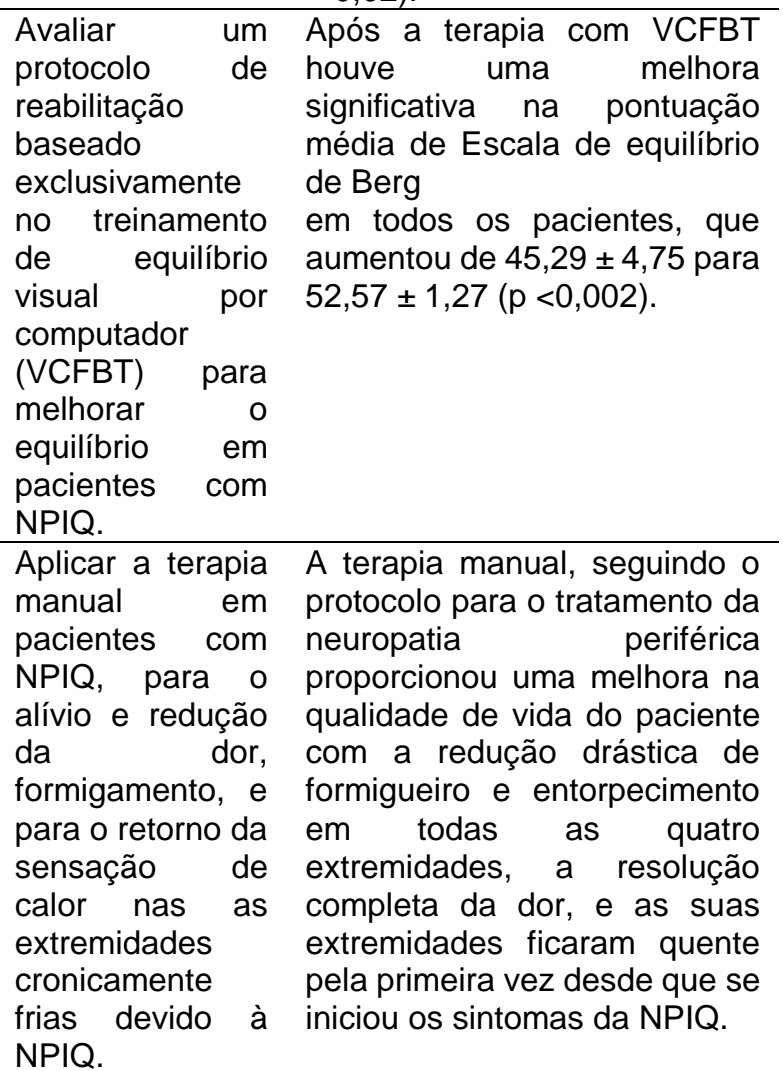
NPIQ. 


\section{DISCUSSÃO}

A NPIQ afeta negativamente tanto a capacidade do paciente de realizar atividades diárias quanto à qualidade de vida relacionada à saúde (QVRS) após o tratamento quimioterápico. Ela manifesta-se como sintomas sensoriais, motores e autonômicos. A neuropatia sensorial é a mais comum, mas os sintomas motores e disfunções autonômicas podem ocorrer.

Os sintomas da neuropatia sensorial incluem dormência, formigamento, queimação das mãos e pés, perda de sensibilidade, parestesia e ocasionalmente dor. Os sintomas motores se apresentam como comprometimento dos nervos motores, fraqueza dos músculos e atrofia muscular. Os sintomas associados à disfunção do sistema nervoso autônomo incluem constipação, hipotensão ortostática e incontinência urinária, devido esses sintomas da neuropatia afetam a qualidade de vida do paciente e podem causar complicações de longa duração (JANG CE, et al., 2018).

Existem alguns recursos não farmacológicos sendo de grande transcendência no manejo dos sintomas de NPIQ, podendo ser introduzido no tratamento como a fisioterapia na qual irá utilizar recursos como massagens e dando ênfase na pratica de exercício físico e acupuntura, abordando assim o conhecimento educativo dos sintomas trazido pelo paciente, envolvendo a família na preparação do ambiente doméstico (SIMÃO DAS, et al., 2015).

No estudo de Zimmer $\mathrm{P}$ et al. (2017) que teve como objetivo avaliar um programa de exercícios com duração de oito semanas, como forma adicional de tratamento para a NPIQ, através de treinos duas vezes por semana, realizando 3 fases que consistiam em: treinos de equilíbrio e coordenação, exercícios aeróbicos com $60 \%$ a $70 \%$ da frequência cardíaca de treinamento e exercícios de fortalecimento muscular global. Dos 30 pacientes, 24 completaram o estudo, onde os sintomas neuropáticos mantiveram-se estáveis no grupo intervenção ao longo do tempo, assim como, observou-se melhora significativa na função de força e equilíbrio, enquanto a NPIQ piorou significativamente no grupo controle.

Segundo Schonsteiner SS et al. (2017), onde ao realizar um ensaio clínico randomizado com o objetivo de avaliar um programa integrado que consistia em mobilizações passivas, exercícios físicos, massagens e a vibração do corpo, realizados em um total de 15 sessões, encontrou-se os seguintes resultados: houve uma melhora significativa em relação ao o contrapeso e a força, uma vez que as melhorias estão fortemente relacionados à $\mathrm{QV}$ dos pacientes.

Os programas de exercícios podem representar uma opção de terapia de suporte promissora para essa população investigação. Um programa de exercícios multimodais pode ser mais eficaz para lidar com uma ampla gama de problemas, incluindo NPIQ.

Cammisuli $S$ et al. (2016) objetivou avaliar um protocolo de reabilitação baseado exclusivamente no treinamento de equilíbrio visual por computador (VCFBT) que melhora o equilíbrio em pacientes com NPIQ, na qual contou com sete participantes, através do uso de um dispositivo de estabilometria estático-dinâmica com capacidade de rotação para medir as forças verticais exercidas pelos pés do paciente e um visual envolvente móvel.

Esse dispositivo fornece a avaliação objetiva e o treinamento do controle motor sensorial e voluntário do equilíbrio através de biofeedback visual em um suporte estável (superfície firme) ou instável (superfície de espuma não rígida).

No presente estudo de Cammisuli $S$ et al. (2016) os protocolos baseiaram-se em exercícios físicos realizados em pé ou caminhando e as performances do sujeito são automaticamente registradas e comparadas com valores de uma população normal, compatível com a idade pelo software do sistema, onde foi proposto um protocolo de reabilitação baseado nos resultados diagnósticos obtidos na entrada (baseline) e os principais comprometimentos do equilíbrio onde elaboraram um protocolo de reabilitação para melhorar a coordenação motora visual dos participantes que foram submetidos a doze sessões de reabilitação de 60 minutos ( 3 vezes / semana durante um período de 4 semanas). Após um mês de reabilitação VCFBT, verificou-se uma melhora clara no equilíbrio. 
Segundo Hagerdorn DK e Holm E (2010) esse tipo de abordagem tem um forte componente de formação de equilíbrio dinâmico, além disso, é um jogo e assim, incentiva a competição entre pacientes que aumenta a adesão no desempenho dos jogos de computador, foi verificado um aumento marcante no resultado de treinamento e aumento de força muscular combinado ao equilíbrio, em consideração aos efeitos limitados nos testes de equilíbrio estático.

Outros recursos terapêuticos são descritos na literatura, como observado no estudo de Cunninggam JE et al. (2011). Neste estudo de caso, os pesquisadores desenvolveram um protocolo baseado na terapia manual em um paciente com NPIQ, para o alívio da dor e do formigamento, e para o retorno da sensação de calor nas extremidades cronicamente frias devido à NPIQ.

Foi realizado em um paciente do sexo masculino, com grau 2 de NPIQ. Após cerca de 8 meses com NPIQ em todas as extremidades (ou seja, cerca de 6 meses após a última quimioterapia), o paciente iniciou um ciclo de 6 semanas de terapia manual administrada em três sessões por semana por dois profissionais licenciados. O tratamento foi administrado como uma combinação de effleurage (acariciando com um toque leve e calmante) e petrissage (amassamento profundo e torcendo em um movimento para baixo e para fora) em cada extremidade.

O tratamento foi aplicado pelos dois terapeutas simultaneamente, primeiro nas extremidades inferiores, logo abaixo dos joelhos, através das pontas dos dedos, e depois nas extremidades superiores, logo abaixo dos cotovelos, através das pontas dos dedos. Antes de cada sessão, o paciente foi solicitado a completar o MD Anderson Symptom Inventory (questionário que avalia a gravidade dos sintomas sentidos pelos pacientes com câncer e a interferência na vida diária causada por esses sintomas) para descrever seus sintomas nas últimas 24 horas em uma escala de 11 pontos, com 0 como não presente e 10 como o pior imaginável. Antes da primeira sessão de terapia manual, ele classificou sua dor como 4 e dormência e formigamento como 10.

Ao final do curso de tratamento, a dor do paciente diminuiu para zero em todas as extremidades. Dormência diminuiu para um zero. Formigamento diminuiu para 1, agora sendo muito leve e apenas em seus dedos, não mais envolvendo palmas ou solas. Suas mãos e pés, que em cada sessão anterior estavam frios ao toque, agora estavam quentes, sem qualquer mudança de roupa ou temperatura externa. Três semanas após o término da terapia manual, seus sintomas de NPIQ permaneceram estáveis e inalterados desde seu último tratamento.

Após mais 3 meses, os sintomas permanecem estáveis nas extremidades superiores. A terapia manual, seguindo o protocolo para o tratamento da neuropatia periférica diabética, quando administrada nas extremidades desse paciente com NPIQ, foi associada a sintomas de NPIQ bastante reduzidos e melhorou acentuadamente a qualidade de vida. Segundo Cassileth BR e Vickers AJ (2004) o toque humano como intervenção contra a dor e outros problemas têm grande artificio.

A massoterapia é definida como manipulação de áreas de tecido mole do corpo e é cada vez mais empregado para alívio dos sintomas em pacientes com câncer. A literatura aponta que a massagem pode diminuir dor em pacientes oncológicos em diferentes estágios da doença.

A massagem terapêutica é um meio não invasivo, de baixo custo e reconfortante, para o controle dos sintomas em pacientes com a doença. Este estudo, portanto, sugere eficácia no tratamento com massoterapia com a melhora dos escores de sintomas dos pacientes oncológicos.

\section{CONSIDERAÇÕES FINAIS}

A NPIQ traz sérias complicações como dor, dormência, parestesia e fraqueza muscular, afetando assim a funcionalidade e consequentemente a qualidade de vida desses pacientes. Há na literatura pesquisas com recursos não farmacológicos tais como: terapia manual associada à massoterapia, treinamento visual de equilíbrio do feedback do computador (VCFBT), um programa de exercícios dinâmicos, aeróbicos e resistidos assim como treinos de equilíbrio e coordenação, para o tratamento e controle da NPIQ. Porém são escassos os estudos que comprovem a eficácia dessas terapias. 


\section{REFERÊNCIAS}

1. BEIJERS AJM, et al. Peripheral neuropathy in colorectal cancer survivors: The influence of oxaliplatin administration. Results from the population-based PROFILES registry. Acta Oncologic, São Paulo, 2015; 54: 463-469.

2. CAMMISULI S, et al. Rehabilitation of balance disturbances due to chemotherapy-induced peripheral neuropathy: a pilot study. Minerva medica, Europe, 2016; 52(4):479-88.

3. CASSILETH BR, VICKERS AJ. Massage Therapy for Symptom Control: Outcome Study at a Major Cancer Center. Journal of Pain and Symptom Management, New York, 2004; V. 28(3): 244-249.

4. CUNNINGHAM JE, et al. Case report of a patient with chemotherapy-induced peripheral neuropathy treated with manual therapy (massage). Suporte Care Cancer, USA, 2011; 19(9): 1473-1476.

5. HAGEDORN DK, HOLM E. Effects of traditional physical training and visual computer feedback training in frail elderly patients. A randomized intervention study. Revista Europeia de Medicina Física e Reabilitação, Europa, $2010 ; 46$ (2): 159-168.

6. JANG CE, et al. The evaluation of changes in peripheral neuropathy and quality-of-life using low-frequency electrostimulation in patients treated with chemotherapy for breast cancer: a study protoco. Trials Jornal Biomed Central, Portugal, 2018; 19:526

7. KLECKNER IR, et al. Effects of exercise during chemotherapy on chemotherapy-induced peripheral neuropathy: a multicenter, randomized controlled trial. Support Care Cancer, EUA, 2018; 26(4): 1019-1028.

8. INSTITUTO DE EVALUACIÓN TECNOLÓGICA EN SALUD. Efectividad y seguridad de gabapentina y pregabalina como monoterapia de primera línea en adultos con dolor neuropático. Bogotá: Colombia; 2013; 59 p.

9. SCHONSTEINER SS, et al. A randomized exploratory phase 2 study in patients with chemotherapy-related peripheral neuropathy evaluating whole-body vibration training as adjunct to an integrated program including massage, passive mobilization and physical exercises. Experimental Hematology \& Oncology, Germany, 2017; 6(5): 1-11.

10. SIMÃO DAS, et al. Chemotherapy-induced peripheral neuropathy: review for clinical practice. Revista dor, São Paulo, $2015 ; 16: 215-220$.

11. TONEZZER T, et al. Effects of transcutaneous electrical nerve stimulation on chemotherapy-induced peripheral neuropathy symptoms (CIPN): a preliminary case-control study. J Phys Ther Sci, São Paulo, 2017; $29(04): 685-692$.

12. VOSGERAU DSR, ROMANOWSKI JP. Estudos de revisão: implicações conceituais e metodológicas. Revista Diálogo Educ, Curitiba, 2014; 14(41): 165-189.

13. XAVIER ATF, et al. Análise de gênero para o adoecer do câncer. Revista brasileira de enfermagem, São Paulo, 2019; 63(6): 921-926.

14. ZIMMER P, et al. Eight-week, multimodal exercise counteracts a progress of chemotherapy-induced peripheral neuropathy and improves balance and strength in metastasized colorectal cancer patients: a randomized controlled trial. Journal Support Care cancer, Germany, 2017; 26(2): 615-624. 